\title{
E3 Ubiquitin-Protein Transferase
}

National Cancer Institute

\section{Source}

National Cancer Institute. E3 Ubiquitin-Protein Transferase. NCI Thesaurus. Code C21254.

An ATP-dependent aminoacyltransferase that catalyzes the third step in the ubiquitination reaction via the transfer of ubiquitin from an E2 ubiquitin-conjug ating enzyme to lysine residues found in the final substrate protein. 